\title{
Single-NUCLEOTIDE POLYMORPHISM IN THE SCCMEC-ORFX JUNCTION DISTINGUISHES BETWEEN LIVESTOCK-ASSOCIATED MRSA CC398 AND HUMAN EPIDEMIC MRSA STRAINS
}

\author{
U Reischl (udo.reischl@klinik.uni-regensburg.de)1 ${ }^{1}$ J Frick ${ }^{2,3}$, S Hoermansdorfer $^{2}$, H Melzl $^{1}$, M Bollwein $^{1}, \mathrm{H}^{\mathrm{J}}$ Linde $^{1}$, \\ K Becker ${ }^{4}$, R Köck ${ }^{4,5}$, C Tuschak², U Busch², A Sing ${ }^{2}$ \\ 1. Institute of Medical Microbiology and Hygiene, University Hospital of Regensburg, Regensburg, Germany \\ 2. Bavarian Health and Food Safety Authority, Oberschleissheim, Germany \\ 3. Clinic for Swine, Veterinary Faculty, Ludwig-Maximilians-University, Oberschleissheim, Germany \\ 4. Institute of Medical Microbiology, University Hospital Münster, Münster, Germany \\ 5. Institute of Hygiene, University Hospital Münster, Münster, Germany
}

This article was published on 10 December 2009.

Citation style for this article: Reischl U, Frick J, Hoermansdorfer S, Melzl H, Bollwein M, Linde HJ, Becker K, Köck R, Tuschak C, Busch U, Sing A. Single-nucleotide polymorphism in the SCCmec-orfX junction distinguishes between livestock-associated MRSA CC398 and human epidemic MRSA strains. Euro Surveill. 2009;14(49):pij=19436. polymorphism in the SCCmec-orfX junction distinguishes between livestock-associatian
Available online: http://www.eurosurveillance.org/ViewArticle.aspx?ArticleId=19436

\begin{abstract}
A number of real-time PCR assays for direct detection of methicillinresistant (MRSA) in clinical specimens are targeting staphylococcal cassette chromosome mec ( $\mathrm{SCCmec}$ ) right extremity sequences and the $S$. aureus chromosomal orf $X$ gene sequences located to the right of the SCC mec integration site. When testing 184 MRSA strains of human and animal origin from geographically distinct locations, we identified several characteristic single-nucleotide polymorphisms (SNPs) within the SCCmec-orfX junction of livestock-associated (LA) MRSA CC398 which serve as suitable strain markers for screening purposes. Within an assay time of 60 minutes and an additional 10 minutes for the melting curve analysis, all MRSA CC398 isolates were correctly identified by their characteristic $T_{m}$ value in the commercial LightCycler MRSA Advanced test. Studies to confirm the diagnostic accuracy of the SNP-based strain identification assay with a larger collection of clinical and LA-MRSA strains are ongoing.
\end{abstract}

\section{Introduction}

Rates of methicillin-resistant Staphylococcus aureus (MRSA) infections have steadily increased during the past two decades and the occurrence and spread of MRSA strains in healthcare facilities as well as in the community is a growing problem worldwide $[1,2,3]$. Although classically considered as a nosocomial pathogen, reports of MRSA carriage or its acquisition in the community have become increasingly common during the past decade $[2,4]$. More recently, studies have demonstrated that especially swine and swine farmers in Austria [5], Denmark [6], Germany [7,8], the Netherlands [9-11], Portugal [12], the United States [13], and many other countries are colonised with MRSA. Since it was realised that livestock animals may form a new, separate reservoir, these strains are now called livestock-associated MRSA (LA-MRSA). Molecular characterisation revealed that a distinct clone of MRSA is predominant within this collective: LA-MRSA strains are grouping within the new clonal complex 398 (CC398) with sequence type 398 (ST398) as the most prevalent type. Animals carrying MRSA represent a reservoir for transmission to humans $[13,14,15]$. The MRSA strains from animal origin have been shown to be pathogenic for humans and can cause severe infections such as endocarditis and ventilator- associated pneumonia [16]. A number of diagnostic strategies have been published on the molecular characterisation of the MRSA CC398 clonal lineage, using pulsed-field gel electrophoresis (PFGE) and multi-locus sequence typing (MLST) or other laborious techniques based on genome sequencing $[1,19,20]$.

\section{Active surveillance is needed}

Since livestock animals may act as a reservoir for this bacterium, the development of rapid molecular methods for screening and identification of MRSA CC398 will have important implications for surveillance, epidemiology and future infection control initiatives. As with many other bacterial pathogens, the rapid and reliable detection of certain MRSA clones is of the utmost importance to prevent the spread of infection. A number of real-time PCR assays targeting staphylococcal cassette chromosome mec (SCCmec) right extremity sequences and the $S$. aureus chromosomal orf $X$ gene sequences located to the right of the SCCmec integration site have recently been established for direct detection of MRSA in clinical specimens. In the course of the present study, such assays were evaluated for their performance to detect and distinguish LA-MRSA strains of the new clonal complex 398 (CC398).

\section{Materials and Methods}

\section{Study population, survey methods and diversity of investigated} strains

During an on-going study conducted by the Bavarian Health and Food Safety Authority to explore the epidemiology of MRSA CC398 in Bavaria, the MRSA colonisation status among swine bred in Bavaria and the involved farmers was investigated by sampling the nares of 634 swine and 116 farmers on 60 geographically distinct farms. Epidemiological results will be available when this particular study is completed. Additional representatives of the MRSA CC398 lineage from other geographic locations and other sources including horses, dogs, guinea pigs, chicken, poultry and humans with contact to colonised animals, as well as MRSA and methicillin-susceptible $S$. aureus (MSSA) isolates of presumed to be related spa-types were kindly provided by a number of supporting laboratories listed in the Table and in the Acknowledgements section. 


\section{Diagnostic culture and template DNA preparation}

The $S$. aureus strains collected from pigs and farmers in Bavaria were maintained on Columbia blood agar and identified by colony morphology, Gram-stain characteristics, catalase reaction, coagulase production, and the results of the API Staph system (bioMérieux). Oxacillin susceptibility was determined by the agar screening method with Mueller-Hinton agar containing $2 \% \mathrm{NaCl}$ and $6 \mathrm{mg} / \mathrm{l}$ of oxacillin for $\mathrm{S}$. aureus [3,19]. An identical protocol was applied for characterisation and maintaining the various $S$. aureus strains provided by supporting laboratories. Template DNA for PCR was prepared from individual bacterial colonies by a simple and rapid 'boiling' procedure [20]. Briefly, colonies were suspended in $200 \mu \mathrm{l}$ of lysis buffer containing $1 \%$ Triton X-100, $0.5 \%$ Tween 20, $10 \mathrm{mM}$ Tris- $\mathrm{HCl} \mathrm{pH} 8.0$ and $1 \mathrm{mM}$ EDTA, and incubated for $10 \mathrm{~min}$ at boiling temperature. After centrifugation for $2 \mathrm{~min}$ at $10.000 \mathrm{x}$ g to sediment the debris, a $2 \mu \mathrm{l}$ aliquot of the clear supernatant was directly transferred to PCR. Alternatively, the reagents and the protocol of the LightCycler Advanced Lysis kit (Roche Diagnostics) were used for template DNA preparation. For cultured bacterial organisms, the efficiencies of the commercial lysis kit and the 'boiling' procedure were found to be comparable for the extraction of amplifiable $S$. aureus DNA (data not shown).

\section{PCR amplification, DNA sequencing and molecular strain typing}

Amplification and sequencing of the SCCmec-orfX junction was performed according to Hagen et al. [21]. PCR reactions and subsequent hybridisation probe melting curve analyses were carried out on a Roche LightCylcer 2.0 device. Amplicons of the expected size were purified (HighPure PCR Cleanup Micro kit,
Roche Diagnostics) and sequenced on an automated $A B I 310$ sequencer using BigDye v. 1.1 chemistry.

Real-time PCR amplification and detection reactions were carried out according to the protocol of the LightCycler MRSA Advanced test (Roche Diagnostics). In the case of a negative result, an in-house duplex PCR assay was performed targeting a segment of the mecA gene and the S. aureus-specific genomic fragment Sa 442 [22]. For selected $S$. aureus strains, accessory testing was performed with well-established commercial PCR tests designed for direct detection of MRSA from clinical specimens, namely the GenoType MRSA Direct (Hain Lifescience), the BD GeneOhm MRSA (Becton Dickinson) and the Xpert MRSA (Cepheid) assays.

The presence of Panton-Valentine leukocidin (PVL) [23] was investigated by PCR testing for the lukS-PVand lukF-PV genes $[2,4]$. Typing of the $S$. aureus protein A gene (spa) was performed for all isolates obtained in this study using a standard protocol [24]. Clustering of spa types into spa clonal complexes (spa-CC) was performed using the Based Upon Repeat Pattern (BURP) algorithm of the Ridom StaphType software (Ridom $\mathrm{GmbH}$ ) with the following preset parameters as recommended previously [25]: Spa types were clustered into the same group if the cost was four or less; spa types which were shorter than five repeats were excluded. When an isolate was indicated to be closely related to a spa type presumed to be associated with CC398, but the $T_{m}$ values observed in the LightCycler MRSA Advanced test did not correspond to the $T_{m}$ values expected for CC398 isolates, MLST- and SCCmec-types of the isolates were determined [1] or provided by the supporting

\begin{tabular}{|c|c|c|c|c|c|c|c|c|c|c|c|}
\hline $\begin{array}{l}\text { Number } \\
\text { of tested } \\
\text { strains }\end{array}$ & MLST & $\begin{array}{l}\text { spa } \\
\text { type }\end{array}$ & $\begin{array}{c}\mathrm{T}_{\mathrm{m}} \\
\text { value } \\
\left({ }^{\circ} \mathrm{C}\right)\end{array}$ & mecA & Sa 422 & $\begin{array}{l}\text { Xpert } \\
\text { MRSA }\end{array}$ & $\begin{array}{c}\text { BD } \\
\text { GeneOhm } \\
\text { MRSA }\end{array}$ & Source & Geographic origin & $\begin{array}{l}\text { Culture } \\
\text { result }\end{array}$ & Comment \\
\hline 8 & ST 398 & to11 & 55 & n.d. & n.d. & pos. & pos. & Piglets & Germany (center 1) & MRSA & Bavarian LA-MRSA survey \\
\hline 11 & ST 398 & t034 & 55 & n.d. & n.d. & pos. & pos. & Piglets & Germany (center 1) & MRSA & Bavarian LA-MRSA survey \\
\hline 11 & ST 398 & to11 & 55 & n.d. & n.d. & pos. & pos. & Fattening pigs & Germany (center 1) & MRSA & Bavarian LA-MRSA survey \\
\hline 5 & ST 398 & t034 & 55 & n.d. & n.d. & pos. & pos. & Fattening pigs & Germany (center 1) & MRSA & Bavarian LA-MRSA survey \\
\hline 5 & ST 398 & t011 & 55 & n.d. & n.d. & pos. & pos. & Humans (pig farmers) & Germany (center 1) & MRSA & Bavarian LA-MRSA survey \\
\hline 4 & ST 398 & t034 & 55 & n.d. & n.d. & pos. & pos. & Humans (pig farmers) & Germany (center 1) & MRSA & Bavarian LA-MRSA survey \\
\hline 1 & ST 398 & t011 & 55 & n.d. & n.d. & n.d. & n.d. & Pig & Germany (center 2) & MRSA & \\
\hline 1 & ST 398 & t2510 & 55 & n.d. & n.d. & n.d. & n.d. & Pig & Germany (center 2) & MRSA & \\
\hline 1 & ST 398 & t1451 & 55 & n.d. & n.d. & n.d. & n.d. & Pig & Germany (center 2) & MRSA & \\
\hline 1 & ST 398 & $\mathrm{t} 108$ & 55 & n.d. & n.d. & n.d. & n.d. & Pig & Germany (center 2) & MRSA & \\
\hline 13 & ST 398 & t011 & 55 & n.d. & n.d. & n.d. & n.d. & $\begin{array}{c}\text { Human (pig farmers } \\
\text { conference) }\end{array}$ & Austria (center 3) & MRSA & Sccmec type V \\
\hline 1 & ST 398 & t2576 & 55 & n.d. & n.d. & n.d. & n.d. & $\begin{array}{c}\begin{array}{c}\text { Human (veterinarian } \\
\text { conference) }\end{array} \\
\end{array}$ & $\begin{array}{c}\text { Austria, Germany, } \\
\text { Switzerland (center 3) }\end{array}$ & MRSA & SCCmec type $V$ \\
\hline 7 & ST 398 & t011 & 55 & n.d. & n.d. & n.d. & n.d. & $\begin{array}{c}\text { Human (veterinarian } \\
\text { conference) }\end{array}$ & $\begin{array}{c}\text { Austria, Germany, } \\
\text { Switzerland (center 3) }\end{array}$ & MRSA & SCCmec type V \\
\hline 3 & ST 398 & t034 & 55 & n.d. & n.d. & n.d. & n.d. & $\begin{array}{c}\begin{array}{c}\text { Human (veterinarian } \\
\text { conference) }\end{array} \\
\end{array}$ & $\begin{array}{c}\text { Austria, Germany, } \\
\text { Switzerland (center 3) }\end{array}$ & MRSA & ScCmec type $\mathrm{V}$ \\
\hline 13 & ST 398 & t011 & 55 & n.d. & n.d. & n.d. & n.d. & $\begin{array}{l}\text { Human (pig farmers } \\
\text { and veterinarians) }\end{array}$ & Austria (center 3) & MRSA & SCCmec type V \\
\hline 24 & ST 398 & to11 & 55 & n.d. & n.d. & n.d. & n.d. & $\begin{array}{c}\text { Dust (from pig } \\
\text { breeding facilities) }\end{array}$ & Austria (center 3) & MRSA & \\
\hline
\end{tabular}




\begin{tabular}{|c|c|c|c|c|c|c|c|c|c|c|c|}
\hline 1 & ST 398 & t034 & 55 & n.d. & n.d. & n.d. & n.d. & $\begin{array}{c}\text { Dust (from pig } \\
\text { breeding facility) }\end{array}$ & Austria (center 3) & MRSA & \\
\hline 10 & ST 398 & t011 & 55 & n.d. & n.d. & n.d. & n.d. & Pigs & Germany (center 8) & MRSA & \\
\hline 1 & ST 398 & to11 & 55 & n.d. & n.d. & n.d. & n.d. & Human (wound swab) & Germany (center 1) & MRSA & \\
\hline 1 & ST 398 & t1456 & 55 & n.d. & n.d. & n.d. & n.d. & Human (wound swab) & Germany (center 1) & MRSA & \\
\hline 1 & ST 398 & t1456 & 55 & n.d. & n.d. & n.d. & n.d. & Human (wound swab) & Germany (center 1) & MRSA & \\
\hline 1 & ST 398 & t011 & 55 & n.d. & n.d. & n.d. & n.d. & Human (nose) & Germany (center 5) & MRSA & \\
\hline 7 & ST 398 & $\mathrm{t} 108$ & 55 & n.d. & n.d. & n.d. & n.d. & Humans & Netherlands (center 7) & MRSA & \\
\hline 2 & ST 398 & t034 & 55 & n.d. & n.d. & n.d. & n.d. & Humans & Netherlands (center 7) & MRSA & \\
\hline 9 & ST 398 & t011 & 55 & n.d. & n.d. & n.d. & n.d. & Humans & Netherlands (center 7) & MRSA & \\
\hline 2 & ST 398 & t034 & 55 & n.d. & n.d. & n.d. & n.d. & Humans & Denmark (center 6) & MRSA & ScCmec type V \\
\hline 1 & ST 398 & t108 & 55 & n.d. & n.d. & n.d. & n.d. & Human & Denmark (center 6) & MRSA & SCCmec type V \\
\hline 2 & ST 398 & t011 & 55 & n.d. & n.d. & n.d. & n.d. & Humans & Denmark (center 6) & MRSA & \\
\hline 1 & ST 398 & t5706 & 55 & n.d. & n.d. & n.d. & n.d. & Human & Denmark (center 6) & MRSA & \\
\hline 1 & ST 398 & t108 & 55 & n.d. & n.d. & n.d. & n.d. & Human & Denmark (center 6) & MRSA & PVL positive, SCCmec type $\mathrm{V}$ \\
\hline 1 & n.d. & t1793 & 55 & n.d. & n.d. & n.d. & n.d. & Human (nose) & Germany (center 2) & MRSA & PVL positive \\
\hline 1 & n.d. & t1250 & 55 & n.d. & n.d. & n.d. & n.d. & Pig & Germany (center 2) & MRSA & \\
\hline 2 & n.d. & t011 & 55 & n.d. & n.d. & n.d. & n.d. & Poultry & Germany (center 2) & MRSA & \\
\hline 1 & n.d. & t011 & 55 & n.d. & n.d. & n.d. & n.d. & Guinea pig & Germany (center 2) & MRSA & \\
\hline 1 & n.d. & t011 & 55 & n.d. & n.d. & n.d. & n.d. & Dog & Germany (center 2) & MRSA & \\
\hline 3 & ST 398 & t011 & 55 & n.d. & n.d. & n.d. & n.d. & Horses & Germany (center 2) & MRSA & \\
\hline 1 & n.d. & t1457 & 55 & n.d. & n.d. & n.d. & n.d. & Human (nose) & Germany (center 2) & MRSA & \\
\hline 1 & n.d. & t1580 & 55 & n.d. & n.d. & n.d. & n.d. & Human (pharynx) & Germany (center 2) & MRSA & \\
\hline 1 & n.d. & t2011 & 55 & n.d. & n.d. & n.d. & n.d. & Human (nose) & Germany (center 2) & MRSA & \\
\hline 1 & n.d. & t1451 & 55 & n.d. & n.d. & n.d. & n.d. & Human (nose) & Germany (center 2) & MRSA & \\
\hline 1 & n.d. & t2346 & 55 & n.d. & n.d. & n.d. & n.d. & Human (nose) & Germany (center 2) & MRSA & \\
\hline 1 & n.d. & t2370 & 55 & n.d. & n.d. & n.d. & n.d. & Human (nose) & Germany (center 2) & MRSA & \\
\hline 1 & n.d. & t2576 & 55 & n.d. & n.d. & n.d. & n.d. & Human (nose) & Germany (center 2) & MRSA & \\
\hline 1 & n.d. & t2741 & 55 & n.d. & n.d. & n.d. & n.d. & Human (nose) & Germany (center 2) & MRSA & \\
\hline 1 & n.d. & t3423 & 55 & n.d. & n.d. & n.d. & n.d. & Human (nose) & Germany (center 2) & MRSA & \\
\hline 1 & n.d. & t1255 & 55 & n.d. & n.d. & n.d. & n.d. & Human (nose) & Germany (center 2) & MRSA & \\
\hline 1 & n.d. & t1197 & 55 & n.d. & n.d. & n.d. & n.d. & Human (nose) & Germany (center 2) & MRSA & \\
\hline 1 & n.d. & t571 & 55 & n.d. & n.d. & n.d. & n.d. & Human (nose) & Germany (center 2) & MRSA & \\
\hline 1 & n.d. & t108 & 55 & n.d. & n.d. & n.d. & n.d. & Human (nose) & Germany (center 2) & MRSA & \\
\hline 1 & n.d. & t2582 & 55 & n.d. & n.d. & n.d. & n.d. & Human (nose) & Germany (center 2) & MRSA & \\
\hline 1 & n.d. & t034 & 55 & n.d. & n.d. & n.d. & n.d. & Human (nose) & Germany (center 2) & MRSA & \\
\hline 1 & ST 398 & t011 & 55 & n.d. & n.d. & n.d. & n.d. & Human (nose) & Germany (center 2) & MRSA & \\
\hline 1 & ST 30 & t138 & 59 & pos. & pos. & pos. & n.d. & Human (nose) & Germany (center 2) & MRSA & \\
\hline 1 & ST 9 & t1430 & 59 & pos. & pos. & pos. & n.d. & Chicken (wing) & Germany (center 4) & MRSA & ScCmec type IVa \\
\hline 1 & ST 1 & t127 & 59 & pos. & pos. & pos. & pos. & Piglet (nose) & Germany (center 1) & MRSA & \\
\hline 1 & ST 398 & t034 & neg. & pos. & pos. & neg. & neg. & Human & Denmark (center 6) & MRSA & SCCmec type IVa \\
\hline 1 & ST 398 & t034 & neg. & pos. & pos. & neg. & neg. & Human & Denmark (center 6) & MRSA & SCCmec type VII \\
\hline 1 & ST 398 & t571 & neg. & pos. & pos. & neg. & neg. & Human & Denmark (center 6) & MRSA & PVL positive, SCCmec non-typeable \\
\hline 1 & ST 398 & $\mathrm{t} 1606$ & neg. & pos. & pos. & neg. & neg. & Human (nose) & Germany (center 2) & MRSA & SCCmec non-typeable \\
\hline 1 & ST 753 & t898 & neg. & pos. & pos. & neg. & neg. & Human (nose) & Germany (center 2) & MRSA & ScCmec non-typeable \\
\hline 1 & ST 398 & t567 & neg. & pos. & pos. & neg. & neg. & Human (nose) & Germany (center 2) & MRSA & SCCmec non-typeable \\
\hline 1 & ST 30 & t021 & neg. & pos. & pos. & neg. & neg. & Human (nose) & Germany (center 2) & MRSA & SCCmec non-typeable \\
\hline
\end{tabular}

BD GeneOhm MRSA: test result of a commercial MRSA-specific PCR assay (Becton Dickinson); MLST: multilocus sequence typing; MRSA: methicillin-resistant Staphylococcus aureus; mecA: test result of an in-house realtime PCR assay targeting the mecA gene [20]; neg: no specific amplification products observed or negative test result for MRSA; n.d. not done; pos: specific amplification products observed or positive test result with the applied realtime PCR assays designed for direct detection of MRSA by targeting the SCCmec-orfX integration site; pSA422: test result of an in-house realtime PCR assay targeting a S. aureus-specific species marker gene Sa422 [20]; $T_{m}: T_{m}$-value observed with the LightCycler MRSA Advanced Test (Roche Diagnostics); Xpert MRSA: test result of a commercial MRSA-specific PCR assay (Cepheid).

MRSA strains were kindly provided by: Bavarian Health and Food Safety Authority, Oberschleissheim, Germany (center 1), University Hospital Münster, Münster, Germany (center 2), B Springer, Austrian Agency for Health and Food Safety, Graz, Austria (center 3), A Fetsch, Federal Institute for Risk Assessment, Berlin, Germany (center 4), J Steinmann, University Hospital Essen, Essen, Germany (center 5), R Skov and J Larsen, Statens Serum Institut, Copenhagen, Denmark (center 6), N Renders, Jeroen Bosch Ziekenhuis, Den Bosch, the Netherlands (center 7), and D Meemken, University of Veterinary Medicine Hannover, Bakum, Germany (center 8). Complete address details are given in the Acknowledgements section. 
Multiple sequence alignment of a selected $S$. aureus orfX segment

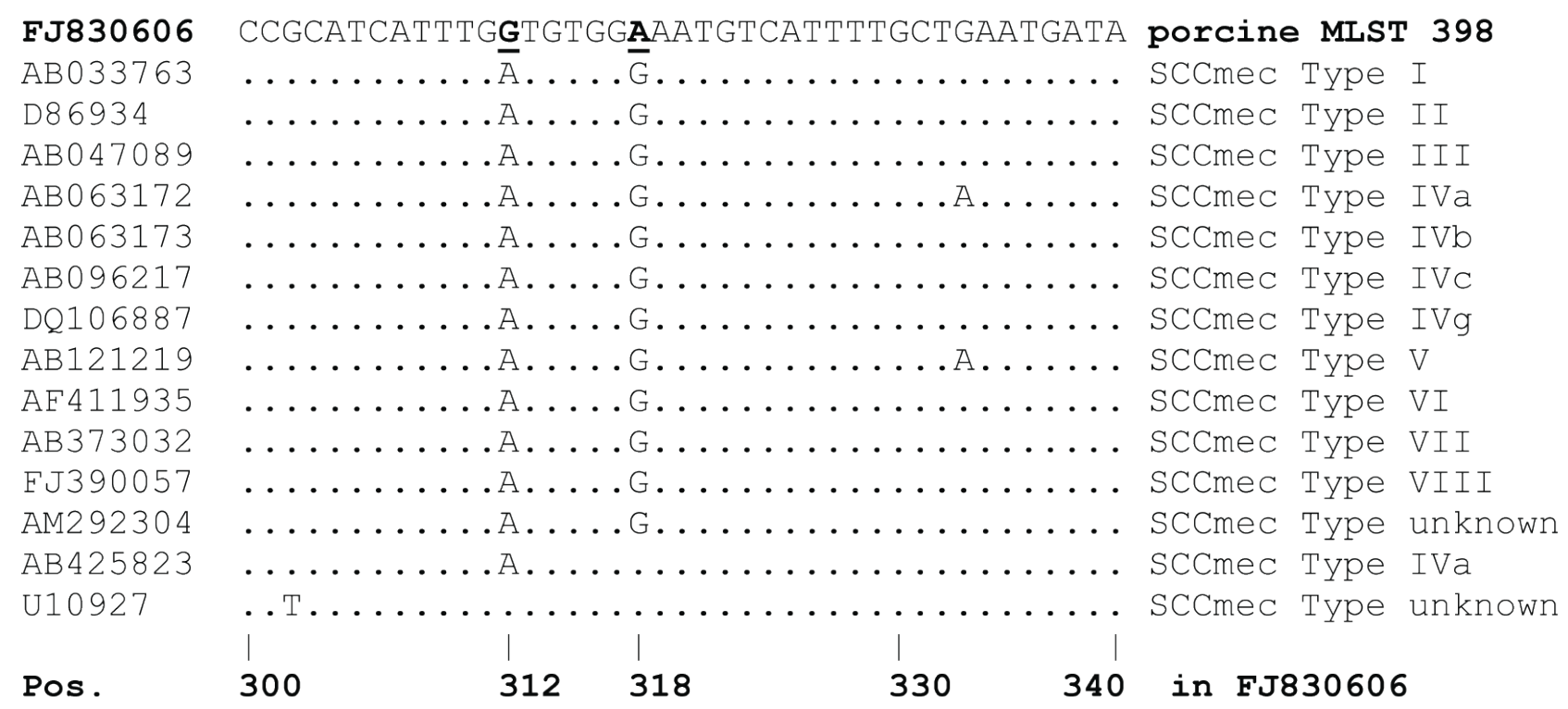

\section{FJ830606}

AB033763

D8 6934

AB0 47089

AB 063172

AB 063173

AB0 96217

DQ106887

AB121219

AF411935

AB373032

FJ390057

AM2 92304

AB 425823

U10927

Pos.

FJ830606

AB 033763

D86934

AB0 47089

AB0 63172

AB 063173

AB0 96217

DQ106887

AB121219

AF 411935

AB373032

FJ390057

AM2 92304

AB 425823

U10927

Pos.

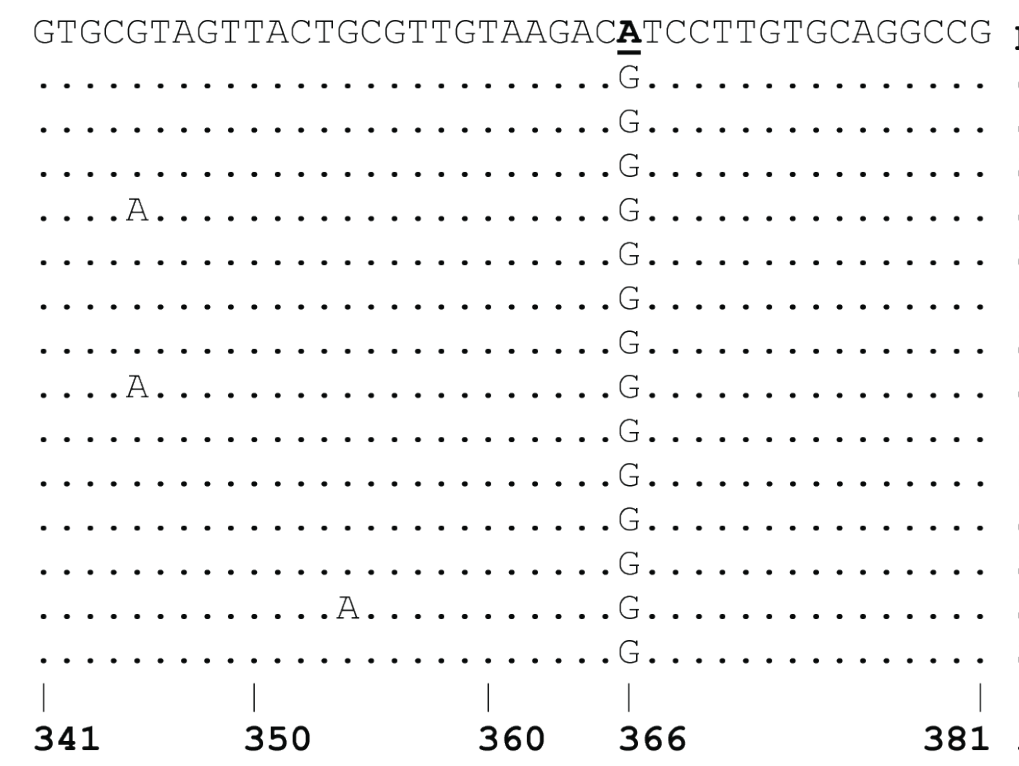

TTTGATCCGCCAATGACGAATACAAAGTCGCTTTGCCCTTG

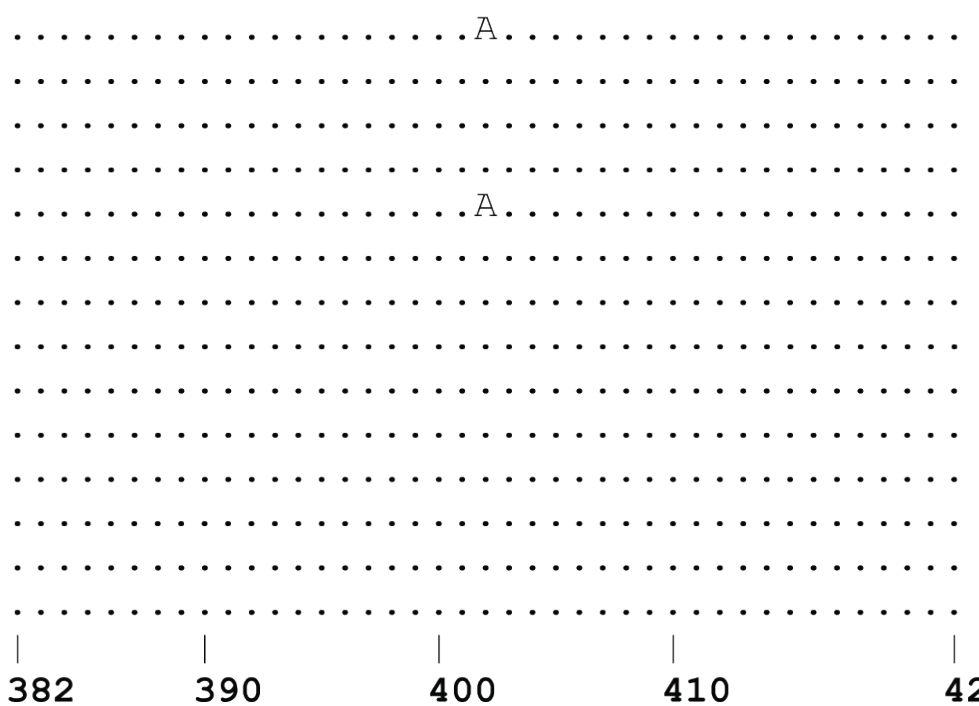

porcine MLST 398

SCCmec Type I

sccmec Type III

ScCmec Type IVa

scCmec Type IVb

ScCmec Type IVC

Sccmec Type IVg

ScCmec Type V

sccmec Type VI

sccmec Type VII

sccmec Type VIII

scCmec Type unknown

sccmec Type IVa

scCmec Type unknown

422 in FJ830606
ScCmec Type II

Multiple sequence alignment of a selected S. aureus orfX segment located close to the SCCmec-orfX junction (position 253 in GenBank FJ830606). The most similar sequences found in BLAST search show either a sequence identical to GenBank sequence entries of $S$. aureus isolates carrying one of the eight SCCmec types or differ from the MRSA ST 398 isolates of the study by at least two nucleotides at positions 312 and 366 (GenBank FJ830606). 
laboratories. Typing of SCCmec elements of types I to VII was carried out according to previously published PCR procedures [26].

\section{Results}

Molecular characteristics of MRSA isolates derived from the

\section{Bavarian LA-MRSA survey}

By sampling the nares of 634 swine and 116 farmers on 60 geographically distinct farms in Bavaria during the course of an ongoing study, a total number of 245 MRSA strains from pigs and 34 MRSA strains from farmers were grown from the collected swabs. From this collection, 44 MRSA isolates from geographically distinct farms were chosen for further analyses (Table, rows 1 to 6 ). The distribution of spa types among these isolates was as follows: t011 $(n=24)$ and t034 $(n=20)$. MLST-typing of all selected MRSA isolates revealed that they belonged to MLST ST398. All 44 MRSA strains tested negative for PVL-encoding genes.

\section{Novel single nucleotide polymorphisms in the SCCmec-orfX} integration site of LA-MRSA isolates

By systematic sequencing of the SCCmec-orfX integration sites of MRSA isolates of animal origin, all of the 44 sequences obtained from Bavarian porcine isolates (Table, rows 1 to 6 ) were found to be identical in a multiple alignment (using pileup from the HUSAR sequence analysis package from the German Cancer Research Center (DKFZ), http://genius.embnet.dkfz-heidelberg.de, data not shown). As the sequence differed from previously published SCCmec-orfX integration site sequence motifs, it was deposited in
GenBank with accession number FJ830606 (to be released after publication).

A detailed investigation of the novel sequence revealed some nucleotide positions uncommon in $S$. aureus GenBank sequences and at least three characteristic single nucleotide polymorphisms (SNPs) in the $S$. aureus chromosomal orfX gene sequence located to the right of the SCCmec integration site: guanine at position 312, adenine at position 366, and cytosine at position 441 (GenBank FJ830606). At least two of these SNPs were found exclusively in the investigated MRSA strains of animal origin and may serve as a diagnostic marker for the presence of MRSA CC398. A BLAST search (National Center for Biotechnology Information (NCBI), http://blast.ncbi.nlm.nih.gov/Blast.cgi) with the complete amplicon sequence revealed GenBank accession number AM292304 ( $S$. aureus SCCmecZH47 mobile element) as the most similar hit with five mismatches. GenBank AB425823 and U10927, the next similar sequences found in the BLAST search, were either identical to GenBank entries of one of the eight acknowledged MRSA SCCmec types deposited in GenBank, or had at least one nucleotide difference at position 366 compared with the sequence FJ830606 obtained from the investigated MRSA ST398 strains of porcine origin (Figure 1).

\section{Practical application of the identified single nucleotide} polymorphisms

In addition to the broad spectrum of unpublished in-house PCR protocols, also the proprietary sensor hybridisation probe of

\section{F I G U R E 2}

Specificity of the Roche LightCycler MRSA Advanced test for differentiating MRSA CC398 and non-CC398 strains in hybridisation probe melting curve analysis

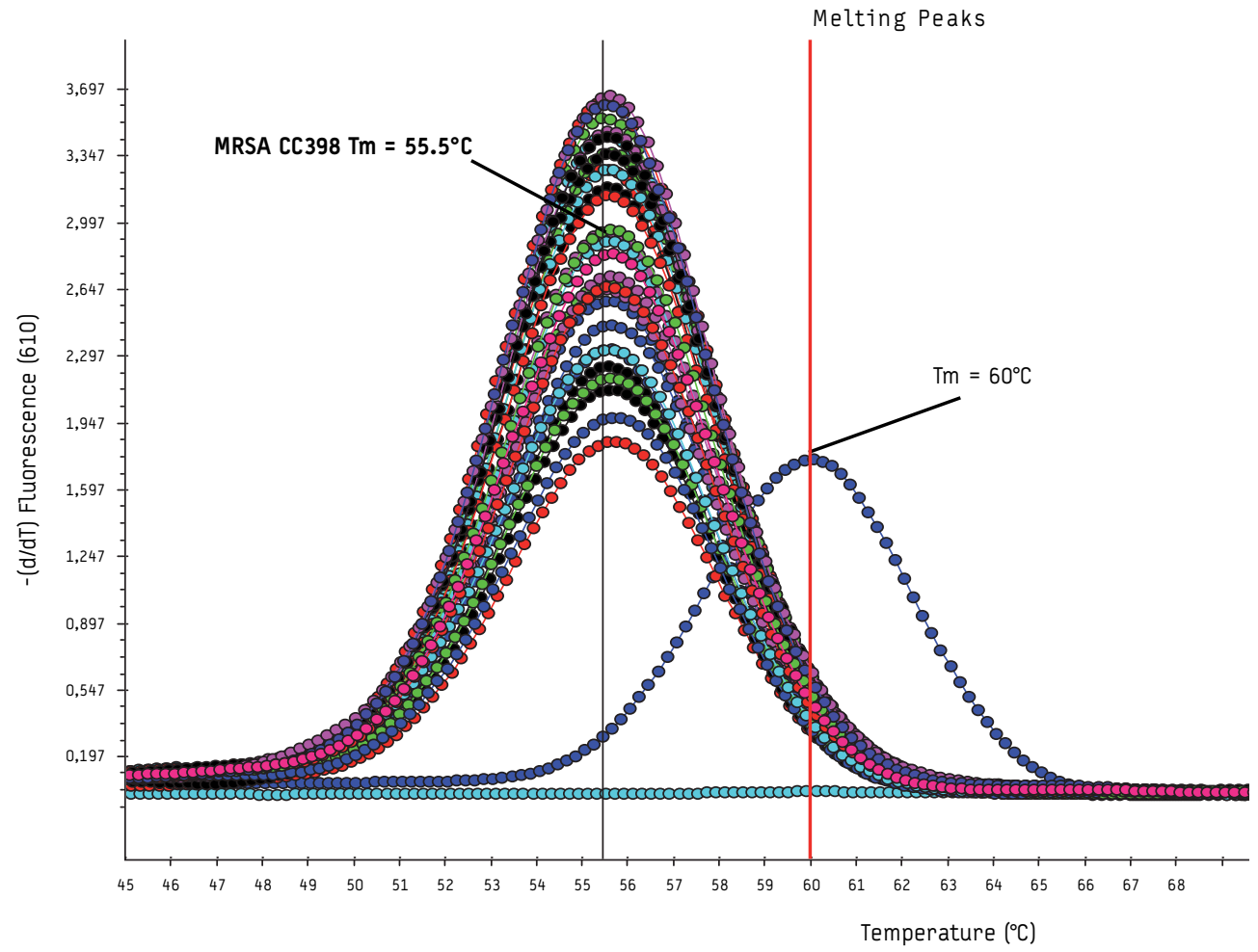

The curves represent the MRSA strain ATCC $33592\left(T_{m} \approx 60^{\circ} \mathrm{C}\right)$ and 30 MRSA ST 398 strains of porcine origin with characteristic Tm values around $55.5^{\circ} \mathrm{C}$. A methicillin-sensitive strain of S. aureus (clinical'isolate) was used as negative control. 
the recently developed LightCycler MRSA Advanced Test (Roche Diagnostics) covers one of these two particular nucleotide positions. This real-time PCR assay detects MRSA strains with different molecular sequences surrounding the right extremity junction of the SCCmec cassette with the S. aureus orfX gene. As a practical application of the SNPs identified in our study, we present the use of this commercial real-time PCR kit for the direct detection of MRSA and simultaneous identification of LA-MRSA CC398. For all 44 investigated Bavarian MRSA ST398 strains, specific amplification products were generated with the LightCycler MRSA Advanced test, and they all harboured at least one of the identified SNPs in the SCCmec-orfX junction represented by a characteristic $\mathrm{T}_{\mathrm{m}}$ of $55.5^{\circ} \mathrm{C}$ in the subsequent LightCycler hybridisation probe melting curve analysis (Figure 2). Since we have not yet observed such a Tm-shift with any non-ST398 MRSA strains of human or animal origin, this point mutation may serve as a molecular marker for the presence of MRSA CC398.

As an approved in vitro diagnostics (IVD) product, the Roche LightCycler MRSA Advanced test has already been validated with a comprehensive collection of MRSA strains of human origin for the limit of detection, inclusivity and exclusivity. The results of systematic studies on the assay's diagnostic performance will be published soon (personal communication, Roche Diagnostics). According to the product information of the test kit, the range of $T_{m}$ values observed in these multicenter validation studies with various epidemic MRSA clones of human origin was from 57.0 to 62.0 ${ }^{\circ} \mathrm{C}$. Therefore a $T_{m}$ value of $55.5{ }^{\circ} \mathrm{C}$ observed with MRSA CC398 should be discriminative with respect to most of the clinical MRSA strains, and melting curve analysis represents a reliable surrogate marker for screening purposes.

From a technical point of view, it should be noted that melting points outside the expected range of 57.0 to $62.0{ }^{\circ} \mathrm{C}$ have to be examined manually in the LightCycler software. When testing MRSA CC398 strains of the present study, the calculation algorithms embedded in the automated assay interpretation software of the LightCycler MRSA Advanced test (Micro Analysis Software; MAS) reported "MRSA result: not detected" with a specific comment "Peak(s) outside Target TM range".

F I G U R E 3

Population snapshot of the tested isolates based on BURP analysis $(n=127)$

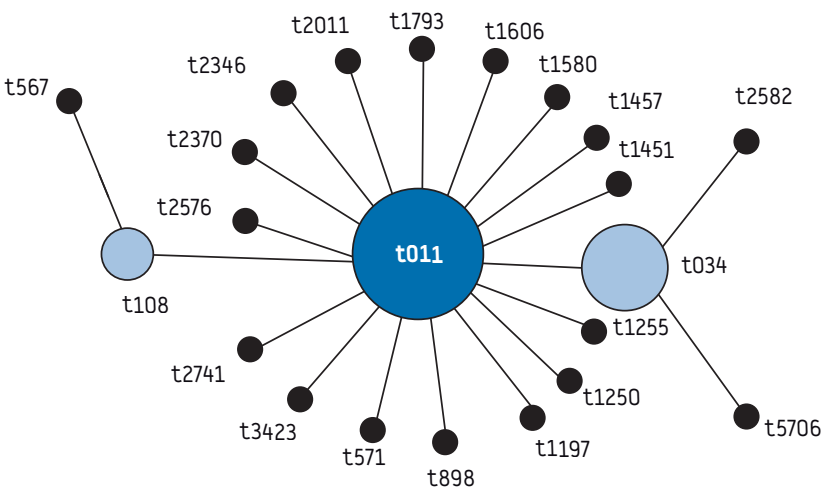

Each dot represents a single spa type and the diameter of the dot reflects the number of isolates associated with the respective spa type. Group founders are coloured in blue, subgroup founders are coloured in light blue.
In the course of the study, we also applied a number of other commercial PCR tests targeting the SCCmec-orfX junction. These included the GenoType MRSA Direct (results not shown), the BD GeneOhm MRSA, and the Xpert MRSA test. The 44 investigated Bavarian MLST CC398 strains, which had all tested positive in the LightCycler MRSA Advanced test, also tested positive for MRSA in these other assays (see Table) - but these PCR test platforms either did not have an option to perform a hybridization probe melting curve analysis or did not allowviewing such melting curve data. Since clinical sensitivity of real-time PCR assays may also depend on the annealing temperatures of the respective probes, it is currently unclear whether the point mutations in the target region will have an impact on the sensitivity when testing samples from patients or animals.

\section{Testing of non-Bavarian MRSA strains within or related to MLST} CC398

In addition to the 44 strains of the Bavarian porcine LA-MRSA survey, 140 MRSA strains recovered from animals and humans in other geographical regions or from other animal sources as well as $S$. aureus isolates of spa-types sharing similar spa repeat patterns, were included in the present study to further address the diversity among isolates within the MLST CC398 clonal complex. Overall, 133 of the 140 isolates were successfully detected by the LightCycler MRSA Advanced test. The collection of investigated strains is shown in detail in the Table, together with the characteristic $T_{m}$ values observed in the LightCycler MRSA Advanced test and the corresponding results of supplementary S. aureus- and mecA-specific PCR assays, as well as the results obtained in other commercial PCR tests targeting the SCCmec-orfX junction. While seven isolates were not detectable, 130 isolates were associated with $T_{m}$ values of $55.5^{\circ} \mathrm{C}$ in the LightCycler MRSA Advanced test, indicative of the presence of the novel SNPs, and three isolates were associated with $T_{m}$ values of $59{ }^{\circ} \mathrm{C}$, known to be within the range observed for the epidemic MRSA clones of human origin.

All of the applied real-time PCR assays, which are designed for direct detection of MRSA by targeting the SCCmec-orfX integration site, failed to generate specific amplification products with seven (3.8\%) of the investigated MRSA strains (Table). The MRSA phenotype of these strains was confirmed by diagnostic culture including oxacillin susceptibility testing. In addition, the MRSA genotype was confirmed by an in-house duplex PCR assay targeting the mecA gene and a S. aureus-specific species marker. SCCmec typing of these seven isolates revealed that one was associated with SCCmec IVa, one with SCCmec VII and five isolates were not typeable using a common SCCmec typing approach.

A population snapshot based on the BURP algorithm was performed for all MRSA isolates included in the study (Figure 3). For arithmetical reasons, three isolates characterised by a $T_{m}$ of $55.5^{\circ} \mathrm{C}$ (two t1456 isolates and one t2510 isolate, all typed as MLST ST398) were excluded from spa cluster formation by BURP because they were shorter than five repeats. The snapshot showed that all remaining 127 isolates associated with $T_{m}$ values of 55.5 ${ }^{\circ} \mathrm{C}$ clustered into one spa-CC. This spa-CC comprised the major spa types t011 and t034 shown to be associated with MLST ST398. This spa-CC contained a further 20 spa types sharing closely related spa repeat patterns: t108, t567, t571, t898, t1197, t1250, t1255, t1451, t1457, t1580, t1606, t1793, t2011, t2346, t2370, t2576, t2582, t2741, t3423 and t5706. 
Moreover, six of the seven isolates not detected by the LightCycler MRSA Advanced test clustered in this spa-CC. MLST typing revealed that five isolates (two of spa type t034 and one each of types t567, t571 and t1606) were associated with ST398, and one isolate associated with spa type t898 was MLST ST753 (90-35-19-2-20-26-39), which is closely related to ST398 (3-3519-2-20-26-39). Thus, all these six isolates were part of the CC398 complex. The remaining isolate not detected by the LightCycler MRSA Advanced test was associated with spa type t021 (ST30).

Those three strains that were characterised by a $T_{m}$ of 59.0 oC in the LightCycler MRSA Advanced test showed spa types t127 (ST1), t138 (ST30) and t1430 (ST9).

\section{Discussion and conclusions}

Although a number of comprehensive studies have been published on the molecular characterisation and detection of the CC398 clonal MRSA lineage using PFGE, MLST or other techniques based on genome sequencing $[1,16]$, this is the first report on a truly rapid detection and/or screening method for this livestockassociated clonal lineage based on characteristic SNPS within a popular target sequence of MRSA-specific PCR assay.

Here, 184 different LA-MRSA isolates obtained from various geographic regions in several European countries and from different sources including pigs, horses, dogs, guinea pigs, chicken, poultry as well as associated in-contact humans were systematically investigated for a characteristic SNP-induced Tm-shift in the LightCycler MRSA Advanced test.

The novel SNPs within the $S$. aureus chromosomal orfX gene detected in the investigated LA-MRSA isolates seemed to represent a conserved sequence motif for these MRSA strains. Even if seven of 184 MRSA strains (six of which were LA-MRSA CC398) were not picked up by the assays due to the presence of uncommon SCCmec elements, it can be stated that the investigated commercial PCR tests targeting the SCCmec-orfX junction showed acceptable inclusivity rates for members of the MRSA CC398 complex. A spa type population snapshot applying the BURP algorithm showed that all MRSA isolates characterised by the SNP-induced Tm-shift in the LightCycler MRSA Advanced test clustered into a distinct spa clonal complex indicative for CC398. Therefore, the novel SNPs within the $S$. aureus chromosomal orf $X$ gene sequences could serve as a discriminative marker for MRSA belonging to the CC398 complex.

It is a well known fact that primer and probe sequences of the current PCR assay concepts are designed to cover the most common $\mathrm{SCC}$ mec types encountered in clinical MRSA isolates. With our increasing knowledge about the enormous sequence diversity of SCCmec sequences, rational primer selection and assay design can only be a best compromise between the coverage of as many SCCmec variants as possible and loss of analytical sensitivity due to primer multiplexing problems in the PCR reaction mixture.

In the course of the present study, we identified a powerful additional feature of the commercial Roche LightCycler MRSA Advanced test. This observation is another example for the fact that the natural diversity of MRSA is also reflected on genomic level. The more isolates are tested for a given target sequence, the more nucleotide mutations or deletions may be encountered. This fact has also implications on the design of specificity panels when developing assays. The assay panel covering epidemiologically relevant clones frequently encountered in patients at risk for MRSA infection is not necessarily congruent with the spectrum of variant isolates that may be found in a specific geographical or epidemiological setting (e.g. introduction of LA-MRSA lineages into a hospital setting). A recent study by Bartels et al. [25] highlighted this problem reporting on a variant SCCmec type IVa clone (spa t024 ST 8) circulating in Copenhagen, which was not detected by a commercial real-time PCR assay targeting the SCCmec-orfX junction.

Now that characteristic SNPs have been identified, colleagues may verify our findings with their collections of animal-associated MRSA strains and may check the primer and probe sequences of their individual in-house PCR protocols targeting the SCCmec-orfX junction for the ability to cover and/or to discriminate MRSA CC398 from human MRSA clones.

If the LightCycler MRSA Advanced test was implemented in a diagnostic laboratory for the intended purpose of direct detection of MRSA in clinical specimens, the occurrence of presumptive MRSA CC398 strains could be monitored without extra work or extra cost just by looking at the melting curve screen. In combination with the simple 'boiling'-protocol for template DNA preparation, it can be easily integrated into the workflow of any clinical or veterinary laboratory routinely using molecular techniques for diagnostic purposes. Once growth of staphylococci is observed on agar plates, a portion of the colony can be transferred to PCR and discriminative MRSA results can be available within 80 minutes. Moreover, knowing about our study results, users of this assay will no longer be confused by the comment "Peak(s) outside Target TM range" generated by the automated assay interpretation software.

In conclusion, the characteristic SNP-induced Tm-shift found in the LightCycler MRSA Advanced test was shown to be suitable to rapidly identify LA-MRSA CC398 clones. By simultaneous screening for general MRSA carriage as well as for MRSA CC398 carriage, this commercial real-time PCR test or comparable assay designs may help to monitor the spread of MRSA CC398 in the human population and, in particular, its importation into healthcare settings. Moreover, this approach may be helpful in screening for MRSA CC398 carriage among animals, farmers or other risk groups.

\section{Acknowledgements}

We gratefully acknowledge the following colleagues for kindly sharing a collection of their valuable and well-characterised LA-MRSA strains, their constructive support and for helpful discussions in the course of the presented study: B Springer, Austrian Agency for Health and Food Safety, Institute of Medical Microbiology and Hygiene, National Reference Laboratory for Antimicrobial Resistance and National Reference Laboratory for coagulase-positive Staphylococci including Staphylococcus aureus, Graz, Austria; A Fetsch, Federal Institute for Risk Assessment - Department "Biological Safety", National Reference Laboratory for coagulase-positive Staphylococci, Berlin, Germany; AW Friedrich, Institute of Hygiene, University Hospital Münster, Münster, Germany; J Steinmann, Institute of Medical Microbiology, University Hospital Essen, Essen, Germany; D Meemken, Field Station for Epidemiology, University of Veterinary Medicine Hannover, Bakum, Germany; R Skov and J Larsen, Staphylococcus Laboratory, National Center for Antimicrobials and Infection Control, Statens Serum Institut, Copenhagen, Denmark; and NHM Renders, Institute of Microbiology, Jeroen Bosch Ziekenhuis, Den Bosch, the Netherlands. The authors would like to thank T Holzmann and $\mathrm{H}$ Stockinger for their active support, and gratefully acknowledge the excellent technical assistance of S Förster and J Fräßdorf during the study. 


\section{References}

1. Enright MC, Day NP, Davies CE, Peacock SJ, Spratt BG. Multilocus sequence typing for characterization of methicillin-resistant and methicillin-susceptible clones of Staphylococcus aureus. J Clin Microbiol. 2000;38(3):1008-15.

2. Lina G, Piémont Y, Godail-Gamot F, Bes M, Peter M, Gauduchon V, Vandenesch $\mathrm{F}$, Etienne J. Involvement of Panton-Valentine leukocidin-producing Staphylococcus aureus in primary skin infections and pneumonia. Clin Inf Dis. 1999;29(5):1128-32.

3. Lowy FD. Staphylococcus aureus infections. N Engl J Med. 1998;339(8):520-2.

4. Reischl U, Tuohy MJ, Hall GS, Procop GW, Lehn N, Linde HJ. Rapid detection of Panton-Valentine leukocidin (PVL)-positive Staphylococcus aureus by real-time PCR targeting the lukS-PV gene. Eur J Clin Microbiol Inf Dis. 2007;26(2):131-5.

5. Springer B, Orendi U, Much P, Höger G, Ruppitsch W, Krziwanek K, et al. Methicillin-resistant Staphylococcus aureus: a new zoonotic agent? Wien Klin Wochenschr. 2009;121(3-4):86-90.

6. Lewis HC, Mølbak K, Reese C, Aarestrup FM, Selchau M, Sørum M, et al. Pigs as source of methicillin-resistant Staphylococcus aureus CC398 infections in humans, Denmark. Emerg Infect Dis. 2008;14(9):1383-9.

7. Meemken D, Cuny C, Witte W, Eichler U, Staudt R, Blaha T. [Occurrence of MRSA in pigs and in humans involved in pig production-preliminary results of a study in the northwest of Germany]. [Article in German]. Dtsch Tieraerztl Wochenschr. 2008;115(4):132-9.

8. Köck R, Harlizius J, Bressan N, Laerberg R, Wieler LH, Witte W, et al. Prevalence and molecular characteristics of methicillin resistant Staphylococcus aureus (MRSA) among pigs on German farms and import of livestock related MRSA into hospitals. Eur J Clin Microbiol Infect Dis. 2009;28(11):1375-82.

9. De Neeling AJ, van den Broek MJ, Spalburg EC, van Santen-Verheuvel MG, DamDeisz WDC, et al. High prevalence of methicillin resistant Staphylococcus aureus in pigs. Vet Microbiol 2007;122(3-4):366-72.

10. van Belkum A, Melles DC, Peeters JK, van Leeuwen WB, van Duijkeren E, Huijsdens XW, et al. Methicillin-resistant and -susceptible Staphylococcus aureus sequence type 398 in pigs and humans. Emerg Infect Dis. 2008;14(3):47983.

11. van Duijkeren E, Ikawaty R, Broekhuizen-Stins MJ, Jansen MD, Spalburg EJ, de Neeling AJ, et al. Transmission of methicillin-resistant Staphylococcus aureus strains between different kinds of pig farms. Vet Microbiol 2008;126(4):383-9.

12. Pomba C, Hasman H, Cavaco LM, da Fonseca JD, Aarestrup FM. First description of meticillin-resistant Staphylococcus aureus (MRSA) CC30 and CC398 from swine in Portugal. Int J Antimicrob Agents. 2009;34(2):193-4.

13. Smith TC, Male MJ, Harper AL, Kroeger JS, Tinkler GP, Moritz ED, et al Methicillin-resistant Staphylococcus aureus (MRSA) strain ST398 is present in midwestern U.S. swine and swine workers. PLoS One. 2008;4(1):e4258.

14. Denis O, Suetens C, Hallin M, Catry B, Ramboer I, Dispas M, et al. Methicillinresistant Staphylococcus aureus ST398 in swine farm personnel, Belgium. Emerg Infect Dis. 2009;15(7):1098-101.

15. Krziwanek K, Metz-Gercek S, Mittermayer H. Methicillin-Resistant Staphylococcus aureus ST398 from human patients, upper Austria. Emerg. Infect. Dis. 2009; 15:766-769.

16. Witte W, Strommenger B, Stanek C, Cuny C. Methicillin-resistant Staphylococcus aureus ST398 in humans and animals, Central Europe. Emerg Infect Dis. 2007;13(2):255-8.

17. Kehrenberg C, Cuny C, Strommenger B, Schwarz S, Witte W. Methicillinresistant and -susceptible Staphylococcus aureus strains of clonal lineages ST398 and ST9 from swine carry the multidrug resistance gene cfr. Antimicrob Agents Chemother. 2009;53(2):779-81.

18. Schwartz DC, Cantor CR. Separation of yeast chromosome-sized DNAs by pulsed field gradient gel electrophoresis. Cell 1984;37(1):67-75

19. Kohner P, Uhl J, Kolbert C, Persing D, Cockerill F. Comparison of susceptibility testing methods with mecA gene analysis for determining oxacillin (methicillin) resistance in clinical isolates of Staphylococcus aureus and coagulase-negative Staphylococcus spp. J Clin Microbiol. 1999;37(9):2952-61.

20. Reischl U, Pulz M, Ehret W, Wolf H. PCR-based detection of Mycobacteria in sputum samples using a simple and reliable DNA extraction protocol. Biotechniques. 1994;17(5):844-5.

21. Hagen RM, Seegmüller I, Navai J, Kappstein I, Lehn N, Miethke T. Development of a real-time PCR assay for rapid identification of methicillin-resistant Staphylococcus aureus from clinical samples. Int J Med Microbiol. 2005;295(2):77-86.

22. Wannet WJ, Spalburg E, Heck ME, Pluister GN, Tiemersma E, Willems RJ, et al. Emergence of virulent mehticillin-resistant Staphylococcus aureus strains carrying Panton-Valentine leucocidine genes in the Netherlands. J Clin Microbiol. 2005;43(7):3341-5.

23. Reischl U, Linde $\mathrm{HJ}$, Metz M, Leppmeier B, Lehn N. Rapid identification of methicillin-resistant Staphylococcus aureus and simultaneous species confirmation using real-time fluorescence. J Clin Microbiol. 2000;38(6), 242933.
24. Harmsen D, Claus H, Witte W, Rothgänger J, Claus H, Turnwald D, et al. Typing of methicillin-resistant Staphylococcus aureus in a university hospital setting using a novel software for spa-repeat determination and database management. J Clin Microbiol. 2003;41(12):5442-8.

25. Mellmann A, Weniger T, Berssenbrügge C, Rothgänger J, Sammeth M, Stoye J, et al. Based Upon Repeat Pattern (BURP): an algorithm to characterize the long-term evolution of Staphylococcus aureus populations based on spa polymorphisms. BMC Microbiol. 2007;29;7:98.

26. Oliveira DC, de Lencastre H. Multiplex PCR strategy for the rapid characterization of structural types and variants of the mec element in methicillin resistant isolates of Staphylococcus aureus. Antimicrob Agents Chemother. 2002;46(7):2155-61.

27. Bartels MD, Boye K, Rohde SM, Larsen AR, Torfs H, Bouchy P, et al. A common variant of staphylococcal cassette chromosome mec type IVa in isolates from Copenhagen, Denmark, is not detected by the BD GeneOhm methicillinresistant Staphylococcus aureus assay. J Clin Microbiol. 2009:47(5):1524-7. 